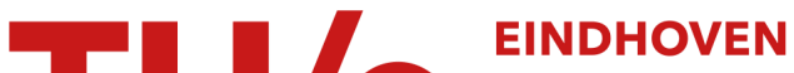 \\ UNIVERSITY OF \\ TECHNOLOGY
}

\section{On final states of two-dimensional decaying turbulence}

Citation for published version (APA):

Yin, Z. (2004). On final states of two-dimensional decaying turbulence. Physics of Fluids, 16(12), 4623-4634. https://doi.org/10.1063/1.1811132

DOI:

10.1063/1.1811132

Document status and date:

Published: 01/01/2004

\section{Document Version:}

Publisher's PDF, also known as Version of Record (includes final page, issue and volume numbers)

\section{Please check the document version of this publication:}

- A submitted manuscript is the version of the article upon submission and before peer-review. There can be important differences between the submitted version and the official published version of record. People interested in the research are advised to contact the author for the final version of the publication, or visit the $\mathrm{DOI}$ to the publisher's website.

- The final author version and the galley proof are versions of the publication after peer review.

- The final published version features the final layout of the paper including the volume, issue and page numbers.

Link to publication

\section{General rights}

Copyright and moral rights for the publications made accessible in the public portal are retained by the authors and/or other copyright owners and it is a condition of accessing publications that users recognise and abide by the legal requirements associated with these rights.

- Users may download and print one copy of any publication from the public portal for the purpose of private study or research.

- You may not further distribute the material or use it for any profit-making activity or commercial gain

- You may freely distribute the URL identifying the publication in the public portal.

If the publication is distributed under the terms of Article $25 \mathrm{fa}$ of the Dutch Copyright Act, indicated by the "Taverne" license above, please follow below link for the End User Agreement:

www.tue.nl/taverne

Take down policy

If you believe that this document breaches copyright please contact us at:

openaccess@tue.nl

providing details and we will investigate your claim. 


\title{
On final states of two-dimensional decaying turbulence
}

\author{
Z. Yin ${ }^{\text {a) }}$ \\ Fluid Dynamics Laboratory, Applied Physics Department, Eindhoven University of Technology, \\ P.O. Box 513, 5600 MB, Eindhoven, The Netherlands and LSEC \& ICMSEC, Academy of Mathematics \\ and System Sciences, Chinese Academy of Sciences, P.O. Box 2719, Beijing 100080, \\ People's Republic of China
}

(Received 6 January 2004; accepted 7 September 2004; published online 9 November 2004)

\begin{abstract}
Numerical and analytical studies of final states of two-dimensional (2D) decaying turbulence are carried out. The first part of this work is trying to give a definition for final states of $2 \mathrm{D}$ decaying turbulence. The functional relation of $\omega-\psi$, which is frequently adopted as the characterization of those final states, is merely a sufficient but not necessary condition; moreover, it is not proper to use it as the definition. It is found that the method through the value of the effective area $S$ covered by the scatter $\omega-\psi$ plot, initially suggested by Read, Rhines, and White ["Geostrophic scatter diagrams and potential vorticity dynamics," J. Atmos. Sci. 43, 3226 (1986)] is more general and suitable for the definition. Based on this concept, a definition is presented, which covers all existing results in late states of decaying 2D flows (including some previous unexplainable weird double-valued $\omega-\psi$ scatter plots). The remaining part of the paper is trying to further study $2 \mathrm{D}$ decaying turbulence with the assistance of this definition. Some numerical results, leading to "bar" final states and further verifying the predictive ability of statistical mechanics [Yin, Montgomery, and Clercx, "Alternative statistical-mechanical descriptions of decaying two-dimensional turbulence in terms of patches and points," Phys. Fluids 15, 1937 (2003)], are reported. It is realized that some simulations with narrow-band energy spectral initial conditions result in some final states that cannot be very well interpreted by the statistical theory (meanwhile, those final states are still in the scope of the definition). (C) 2004 American Institute of Physics. [DOI: 10.1063/1.1811132]
\end{abstract}

\section{INTRODUCTION}

It is an interesting topic to study final states of twodimensional (2D) decaying turbulence. Recent publications ${ }^{1}$ [hereafter YMC (Yin-Montgomery-Clercx)] and Ref. 2 show that numerical simulations starting from different initial conditions (depending on sizes of patches in the vorticity field) can lead to different final states. The statistical mechanics in 2D turbulence, known as the "point theory" and the "patch" theory, reveals a great predictive power in this kind of simulations.

The point theory, advanced 30 years ago, ${ }^{3,4}$ is concerned with a mean-field treatment of ideal line vortices. The system is Hamiltonian with a finite phase space, applied by Boltzmann statistics to its dynamics initially by Onsager. ${ }^{5}$ It was further developed by several groups. ${ }^{6-22}$ In these studies, it is surprising to see that the ideal Euler mean-field predictions fit the Navier-Stokes (NS) results. The patch theory was put forward since the late $1980 \mathrm{~s}^{23-28}$

In the patch theory, the $\delta$ functions used to discretize the vorticity field in the point theory are replaced with finite area, mutually exclusive patches of vorticity. The LyndenBell statistics ${ }^{29}$ is applied to this theory.

The predictive abilities of these two theories are tested after the related entropies of them are defined and the precise formulas suited to calculations developed. These details are provided in Spring Notes by D. C. Montgomery (private communication). Several kinds of solutions in the doubly

a)Electronic mail: yinzh@1sec.cc.ac.cn periodic domain from the statistical mechanics are considered. It is found that the traditional "dipole" or the onedimensional "bar" solution will dominate the final state under different conditions. For the initial vorticity field with large patch vortices, the bar solution will appear finally, and the dipole will dominate the flow field if only point vortices or small patches exist initially. The prediction is validated by the most direct numerical simulations of us, which are well represented by numerical results in YMC. These simulations, which employ Fourier pseudospectral methods with considerable high resolutions $\left(512^{2}\right)$, have been run long enough (100-1500 turnover times) to ensure the reach to final states. We even found a couple of results leading to unclassified states, which are excluded by the most general case of the patch theory (see Secs. II and III in this paper for some results).

However, compared with considerable efforts to investigate the "final state" of 2D turbulence, few efforts have been devoted to defining it. There exist some characteristics when the term of final states in 2D flows is referred to, but they are rather blurred and it is very easy to find some counterexamples for them as the research in this field goes on. For instance, sometimes it is thought that the flow field has reached the final state if the pattern of the flow remains unchanged for a certain long time. However, in our sinhPoisson quadrupole to dipole simulation (Fig. 7 in Ref. 2), the quadrupole in the vorticity field lasts so long (from $t$ $\cong 0$ to $t \cong 150$ ) that people might think the final state has already been reached, but the continued calculation shows 
that it is merely an intermediate state towards the dipolar final state. Besides the above example, some other characteristics look promising to be the definition of the final state in 2D turbulence. Again, we can find some counter examples for these traits after some strict reasoning (we will discuss another one in detail later in Sec. II A). Hence, it is necessary to give a definition that can cover all exiting cases of final states. This definition will play an important role when a long direct numerical simulation of decaying turbulence is carried out. It can be used to decide when the code should be stopped. This is not a trivial decision since some of the results (including the bar final state) in YMC are obtained partly because of the continuing calculation after former researchers stopped ${ }^{30}$ (of course, those runs are mainly stimulated by theoretical results of the statistical mechanics). In our former study, the pseudospectral code has been continued as long as possible to make sure that final states have been reached. The calculation will be enormously extended without a good definition of the final state. On the other hand, owning to the existing bottleneck in parallelization of the $2 \mathrm{D}$ pseudospectral code, ${ }^{2}$ the extended calculation might mean several-extra-weeks CPU time for runs with the resolution of $512^{2}$. The future research in this field may involve simulations with the resolutions of $1024^{2}$ or higher. For those high-resolution simulations, it would be a nightmare for the study if no clue is adopted to judge the final state. A lot of extended computation is required to ensure that there is no more interesting new phenomenon. In Sec. II, we will try to give a definition of final states.

In Sec. III, with the assistance of the definition of final states (on the other hand, in order to validate the definition), some numerical simulations are fulfilled. This part of the work can also be regarded as the continued investigation of YMC. Former numerical results leading to bar final states in YMC are confined by an initial symmetric quadrupole with a certain amount of noise added to break the symmetry and accelerate the process of computations. Without further proofs, it can be argued that the bar is reached only because of the existing symmetry in the main structure. Therefore, the generality of this kind of solutions might be endangered. In Sec. III A, some other techniques are adopted to break the symmetry, and again they end up with bar states.

In Sec. III B, a totally different initial condition bringing about the bar final state will be introduced. Although the statistical mechanics can predict the flow pattern for the final state of that simulation, it has some difficulties in explaining the $\omega-\psi$ scatter plot at the late time. Here, it should be noticed that that final state is still in the scope of the definition in Sec. II B.

There are two main threads when this paper is being worked on: one is the different definition of final states, and the other is that this paper is the extended work of YMC.

\section{DEFINING THE FINAL STATE IN 2D TURBULENCE}

\section{A. Starting from a sufficient but not necessary condition of final states}

In 2D flow field, if we denote the vorticity as $\omega$ and the stream function $\psi$, the NS equation can be written as

$$
\frac{\partial \omega}{\partial t}+\left(\frac{\partial \omega}{\partial x} \frac{\partial \psi}{\partial y}-\frac{\partial \omega}{\partial y} \frac{\partial \psi}{\partial x}\right)=\nu \nabla^{2} \omega
$$

and

$$
\omega=-\nabla^{2} \psi,
$$

with $\nu$ the kinematic viscosity of the fluid. A shorthand notation for the nonlinear contribution to Eq. (1) is the Jacobian $J(\omega, \psi)$,

$$
J(\omega, \psi)=\frac{\partial \omega}{\partial x} \frac{\partial \psi}{\partial y}-\frac{\partial \omega}{\partial y} \frac{\partial \psi}{\partial x} .
$$

For inviscid flows, Eq. (1) reduces to the Euler equation

$$
\frac{D \omega}{D t}=\frac{\partial \omega}{\partial t}+J(\omega, \psi)=0,
$$

which states that the vorticity of a fluid element is conserved for inviscid flows (note that $D \omega / D t$ represents the material derivative).

It can be observed that if there exists a functional relation $\omega=f(\psi)$, then

$$
\begin{aligned}
J(\omega, \psi) & =\frac{\partial f(\psi)}{\partial x} \frac{\partial \psi}{\partial y}-\frac{\partial f(\psi)}{\partial y} \frac{\partial \psi}{\partial x} \\
& =\left(\frac{\partial f}{\partial \psi} \frac{\partial \psi}{\partial x}\right) \frac{\partial \psi}{\partial y}-\left(\frac{\partial f}{\partial \psi} \frac{\partial \psi}{\partial y}\right) \frac{\partial \psi}{\partial x}=0 .
\end{aligned}
$$

This means that the experimental or numerical observation of the functional relationship $\omega=f(\psi)$ indicates the presence of a stationary state of inviscid flow. Usually, this observation is considered as an indication of the presence of the final state of high Reynolds number flows, i.e., the case when $\nu$ $\rightarrow 0$.

Although the $\omega-\psi$ functional relation is an important tool in the characterization of so-called final states of decaying $2 D$ turbulence, it should be noticed that it is a sufficient but not necessary condition of near-equilibrium states of high Reynolds number flows.

In Fig. 1, we see a good example of the functional relationship between $\omega$ and $\psi$-the famous Lamb dipole (for further details see Refs. 31 and 32). It has the linear relation of $\omega-\psi$ (for $-0.5<\psi<0.5$ ). Another example is the so-called $\omega-\psi$ sin-hyperbolic relationship, ${ }^{33-35}$ which normally appears in the late state of 2D decaying turbulence with a broad band energy spectral initial condition.

In Fig. 2, we have a double-valued structure of the $\omega-\psi \operatorname{plot}^{36}$ and the associated $\omega$ and $\psi$ contour plots. They come from one simulation in YMC, Figs. 18 and 19, which cannot be explained by the existing statistical-mechanical theories. With a comparison between the $\omega-\psi$ scatter plot and the contour plots of $\omega$ and $\psi$, it can be concluded that the larger negative vortex indicated by the solid arrow corresponds to the longer negative branch of the $\omega$ - $\psi$ plot. In the meanwhile, the shorter (negative) branch of the $\omega-\psi$ plot represents the smaller negative vortex (see the dashed arrow).

The vorticity field shown in Fig. 2(a) represents a stationary solution of the Euler equation $D \omega / D t=0$. This can be 

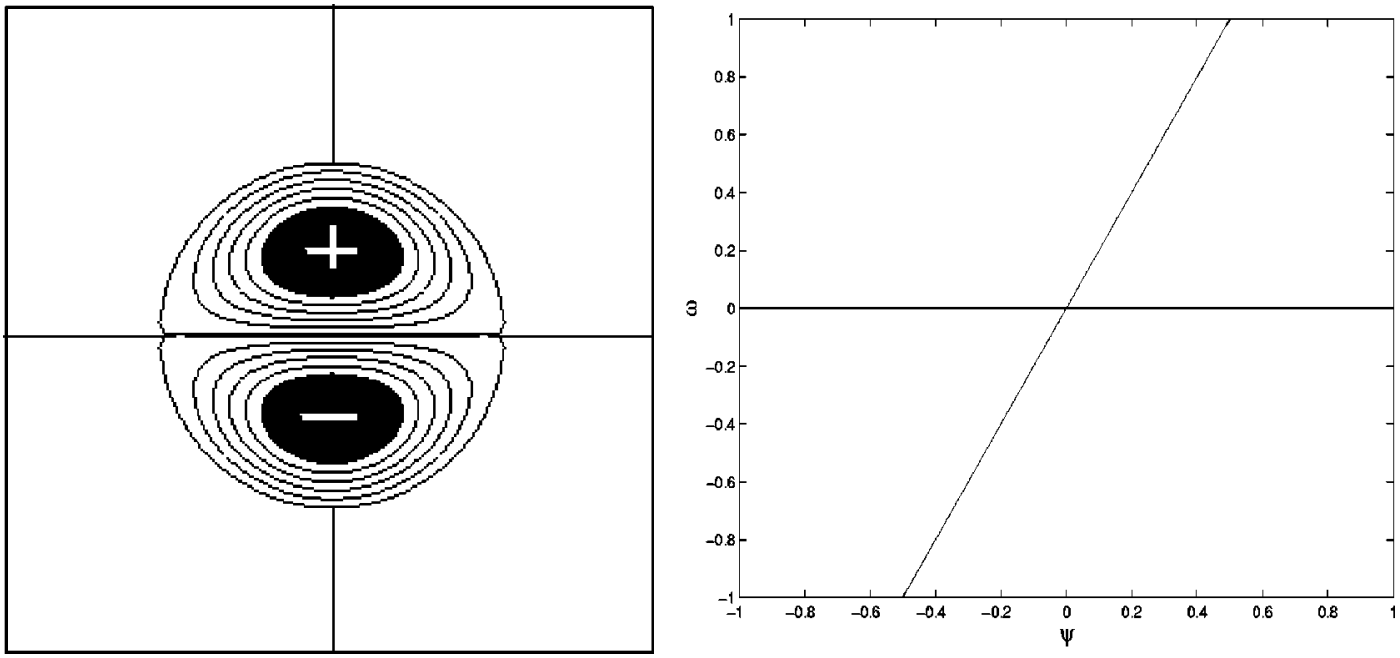

FIG. 1. The Lamb dipole with a linear relationship between $\omega$ and $\psi$. It is a stationary two-dimensional solution of the Euler equation. The figure on the left indicates the vorticity field, with one positive vortex and one negative vortex confined within the circle. Outside that circle, the vorticity is zero.

demonstrated numerically: what we did was to use the pseudospectral Fourier code of the NS equation and setting $\nu$ $=0$ (this is the easiest way to carry out in our case). The exact vorticity field of Fig. 2(a) (without any noise) is taken as the initial condition for a simulation with the resolution of $512^{2}$. The simulation lasts for $3 \times 10^{5}$ time steps (the time step is 0.0005)—about 400 turnover times if we set $\nu=1 / 5000$. However, the vorticity field has not been changed a bit from $t=0$ to $t=150$. The continuing running of the code is simply a test of the accuracy of the pseudospectral method.

This double-valued structure cannot be interpreted by the statistical-mechanical theory for Euler flows, even if the most general formulation of the patch theory
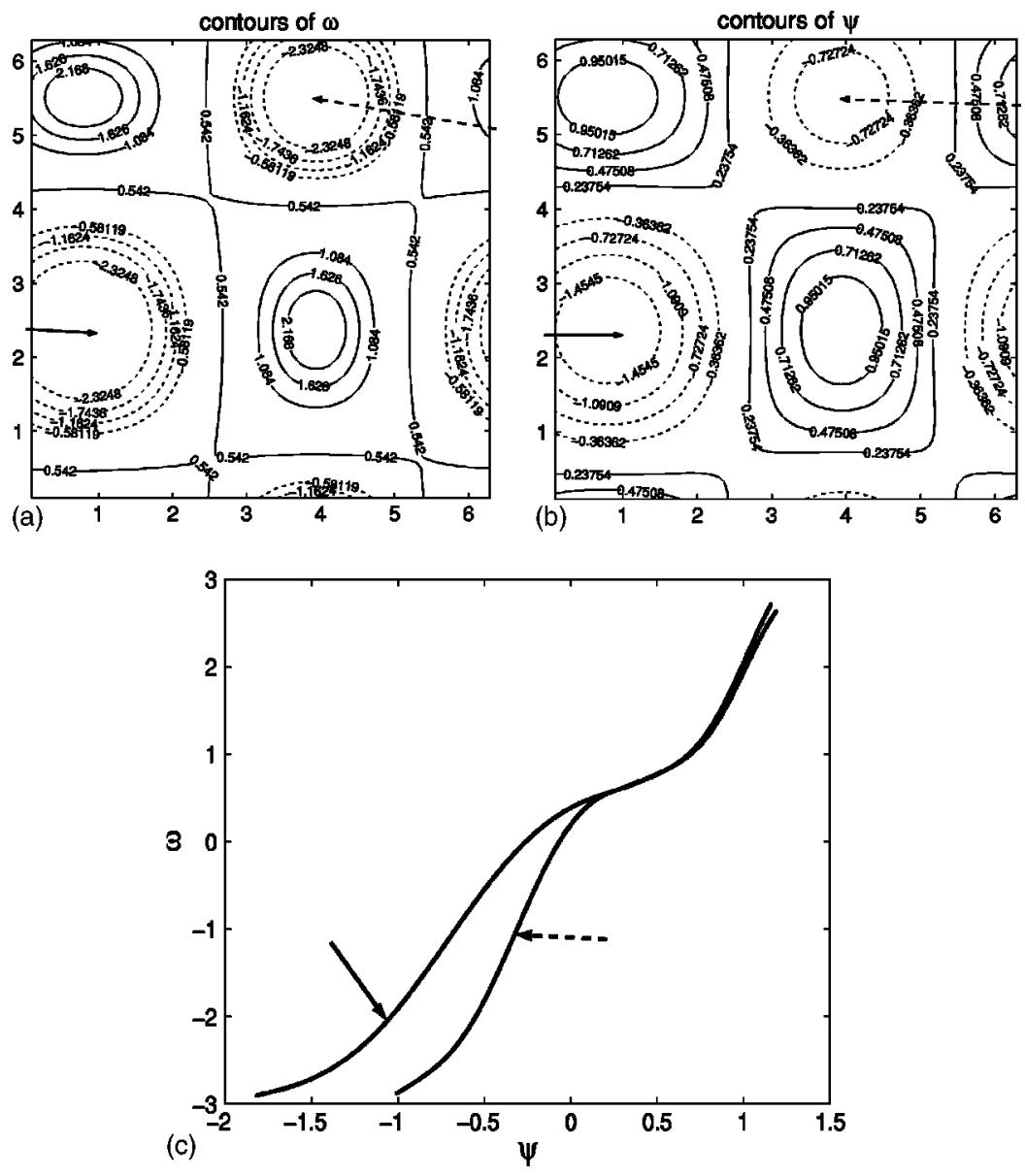

FIG. 2. The double-valued structure, representing a nonfunctional $\omega-\psi$ relation (c), is still a stationary solution of the Euler equation. The associated vorticity and stream function contour plots are shown in (a) and (b). 


$$
\nabla^{2} \psi=-\omega=-\sum_{j=1}^{q} \frac{M}{\Delta} K_{j} \frac{e^{\alpha_{j}-\beta \psi K_{j}}}{\sum_{l=0}^{q} e^{\alpha_{l}-\beta \psi K_{l}}}
$$

is taken into consideration. At this point, we should admit that although the statistical-mechanical theory appears very powerful for the study of freely evolving 2D turbulent flows, it still has some limitations, which cannot be easily understood.

The flow is not described by any functional relation between $\omega$ and $\psi$, but it manages to go to one specific kind of equilibrium state. It is partly because this simulation starts from a condition with narrow-band energy spectra-more specifically, most energy is concentrated in few low wave numbers. Because of inverse energy cascade phenomena in 2D turbulence (the energy is mainly transferred to lower wave numbers), the state of broadband energy spectra, where the statistical mechanics might take effect, can never be reached.

Anyway, the functional relation of $\omega-\psi$ is not enough to define the final state, and something else should be applied as the definition. This will be the main task in the following section.

\section{B. A definition covering all existing possibilities}

To estimate the quantitative flux across the region, people normally employ a diagnostic technique first introduced by Read, Rhines, and White. ${ }^{37}$ They showed that the net flux of vorticity out of a closed loop in the physical space is equal to the effective area enclosed by the corresponding circuit in the $\omega-\psi$ space. However, the usage of the effective area is so far limited to reveal how far the flow field is away from the state of the $\omega-\psi$ functional relation. ${ }^{38,39}$ In the following discussion, we will show that it is in fact a more powerful and more general judgment for final states of $2 \mathrm{D}$ turbulence than the simple $\omega-\psi$ functional relation.

First, a short outline of this diagnostic technique will be provided in order to be self-contained.

(1) At a certain stage of a numerical simulation of 2D decaying turbulence, draw a closed circuit in the contour plot of $\psi$ that can represent the whole flow field, find enough points on this circuit, and mark them in order [Fig. 3(a)]. It is crucial to the whole judgment procedure when this step should be taken to judge the final state. We will discuss it later in this section.

(2) Find the corresponding points in the scatter plot of $\omega-\psi$ at the same time of the simulation [Figs. 3(b) and 3(c)]. The "new" labeled points will also form a closed circuit (or, several closed circuits in the case of a reentrant region). The tags of the points are used to show the directions (clockwise or counterclockwise) of those circuits. In Fig. 3, we only use five points, which are merely enough to indicate the directions of all involved regions (in the contour plot of $\psi$ and in the scatter plot of $\omega-\psi)$.

(3) There will be two kinds of possibilities for the corresponding points in the scatter plot of $\omega-\psi$, which are as follows.

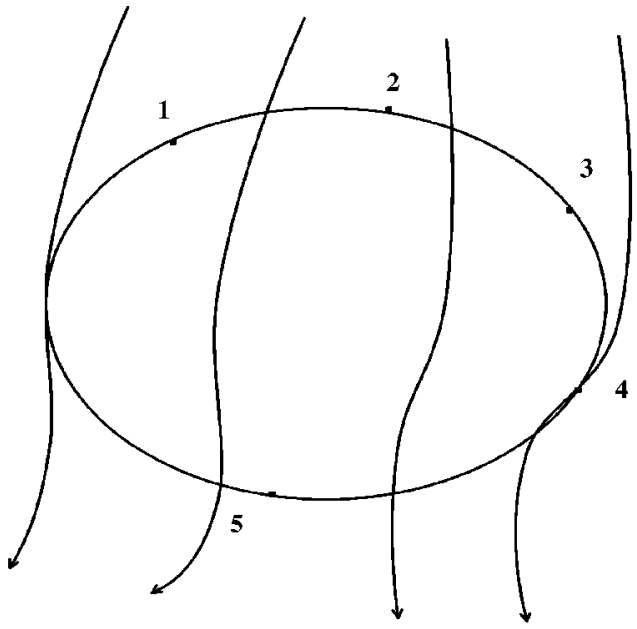

(a)
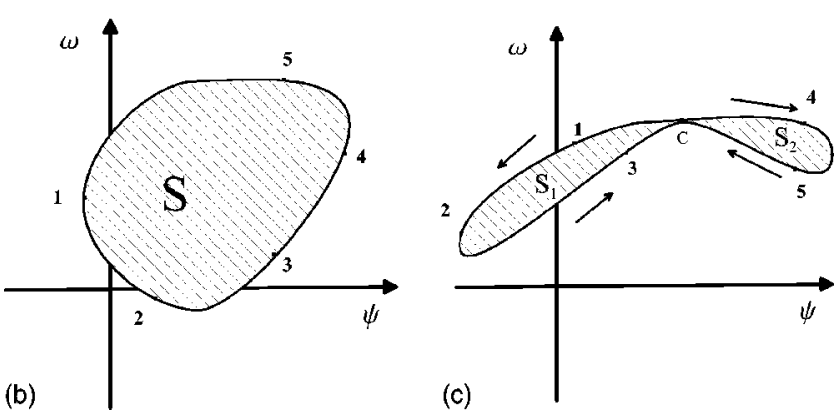

FIG. 3. (a) is the contour plot of the stream function $\psi$ for a flow field, and there is a circuit with five marked points on it; (b) indicates one possible distribution of the five marked points in the $\omega-\psi$ space- they form a simple circuit; (c) suggests another possibility-a reentrant area: $S_{1}$ is the counterclockwise region and $S_{2}$ the clockwise region.

(i) Those points form a simple circuit, the area of which is equal to the effective area $S$ [Fig. 3(b)].

(ii) Those points form a reentrant region. The effective area $S$ in this case is equal to the sum of counterclockwise areas minus the sum of clockwise areas. For example, in Fig. 3(c), the effective area $S$ is

$S=S_{\text {anticlockwise }}-S_{\text {clockwise }}=S_{1}-S_{2}$.

[In Fig. 3(c), we only drew one clockwise and one counterclockwise region for convenience. In practice, it may have several clockwise and counterclockwise areas, respectively.]

(4) The absolute value of $S$ indicates how far the flow field is away from the "near steady" state (not the final state). The larger the absolute value of $S$, the farther the flow field away from the near steady state. On the other hand, if the absolute value of $S$ is very small $(S \cong 0)$, it means that the near steady state has been reached.

(5) The condition of $S \cong 0$ is only enough to judge the near steady state of $2 \mathrm{D}$ turbulence. To define the final state, it is necessary to remove those local equilibrium states (e.g., see the "8-bar" state in Fig. 11 of YMC).

In practice, it is not always easy to find a closed circuit in the contour plot of $\psi$ that can represent the whole flow 

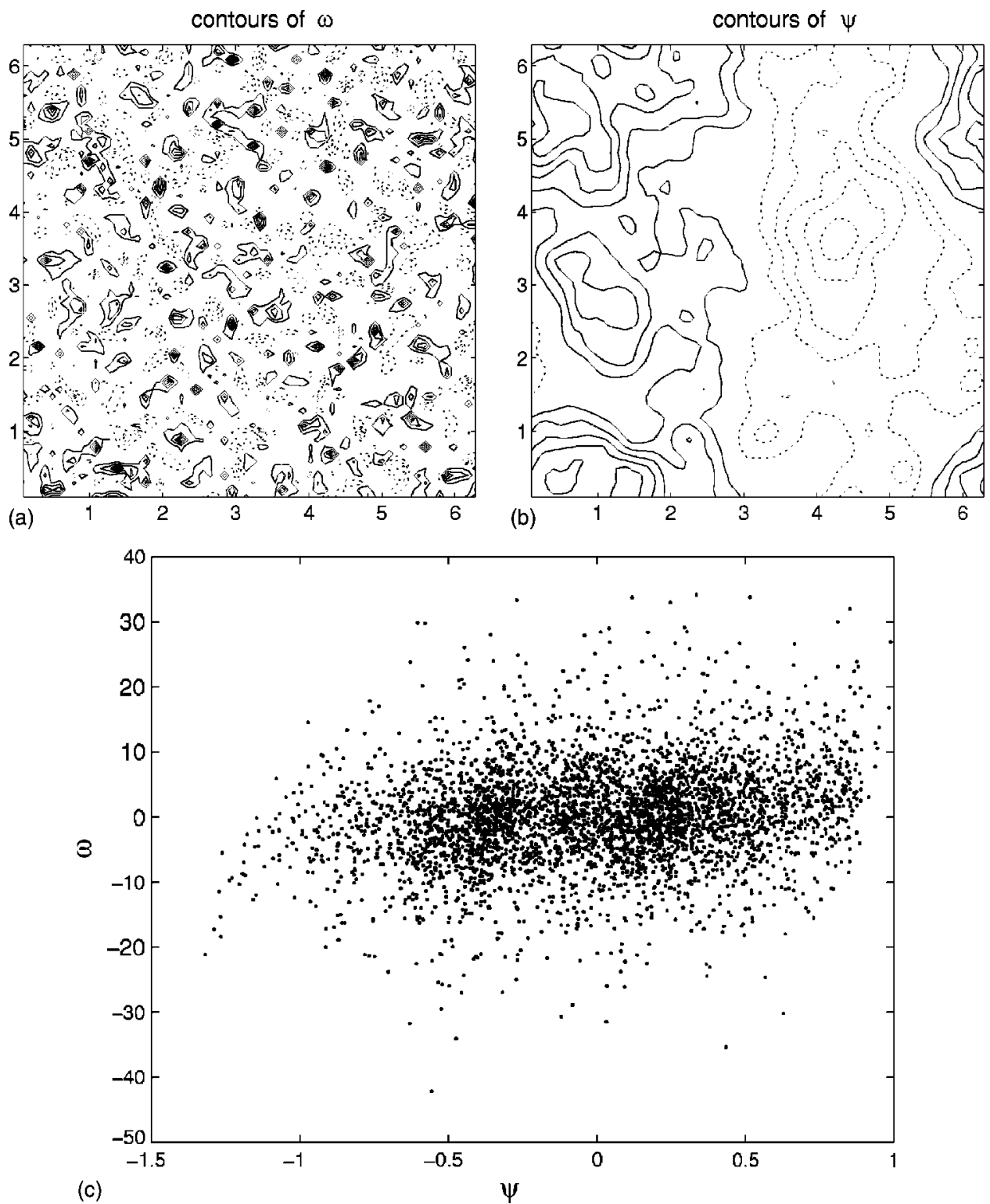

FIG. 4. (a) and (b) are the contour plots of $\omega$ and $\psi$ at the same time of the early stage of a simulation starting from some random noise. It is very hard to find a circuit that can "represent" the whole flow field on (b). (c) is the corresponding $\omega-\psi$ scatter plot, in which no clear structure can be observed. field, especially if we have a very random flow field [e.g., Figs. 4(a) and 4(b)]. To implement the technique discussed above, it is not advisable to judge whether the flow has reached the final state or not by trying to find the "representing" circuit. It is easier to draw the scatter $\omega-\psi$ plot of the flow first - by doing this, all the information we have about the flow field is used. If there is no clear structure on the $\omega$ - $\psi$ plot [e.g., Fig. 4(c)], we know that it is unnecessary to find the representing circuit and continuing computations are needed. In the case that a functional $\omega-\psi$ plot is shown up, the procedure of finding the closed circuit is not necessary at all. So actually, we only need to find the representing circuit in the $\psi$ contour plot when the $\omega-\psi$ plot has a relatively simple multivalued structure. Normally, this should not be a very difficult task because the flow field is already very simple (e.g., see Sec. III B).

To sum up, the final state of 2D turbulence can be defined as the flow field of $2 D$ turbulence has reached the final state if the following two conditions are true: (a) The effective area $S \cong 0$; (b) it is not a local equilibrium state.
Note that the "real" final state of the flow field is the zero vorticity state $(\omega \equiv 0)$ when the decaying process really stops, but those cases do not appeal to us. The final state talked about now is actually a stage when the continuing numerical simulation will not cause any interesting phenomenon. Here, the condition of $S \cong 0$ cannot be strictly defined. How small the absolute value of $S$ should be, depends on different specific conditions. According to our experience, it is more useful to look into the scatter plot of $\omega$ - $\psi$ itself than to give out any specific value.

In the following, the new definition will be used to analyze different situations.

(I) For those results with the functional relation of $\omega-\psi$ such as the Lamb dipole or the "sinh-like" relation discussed in the preceding section, it is obvious that $S \cong 0$ - the "final state" has been reached.

(II) For those results with multivalued structures in $\omega-\psi$ plots, there are three kinds of situations, which are as follows. 

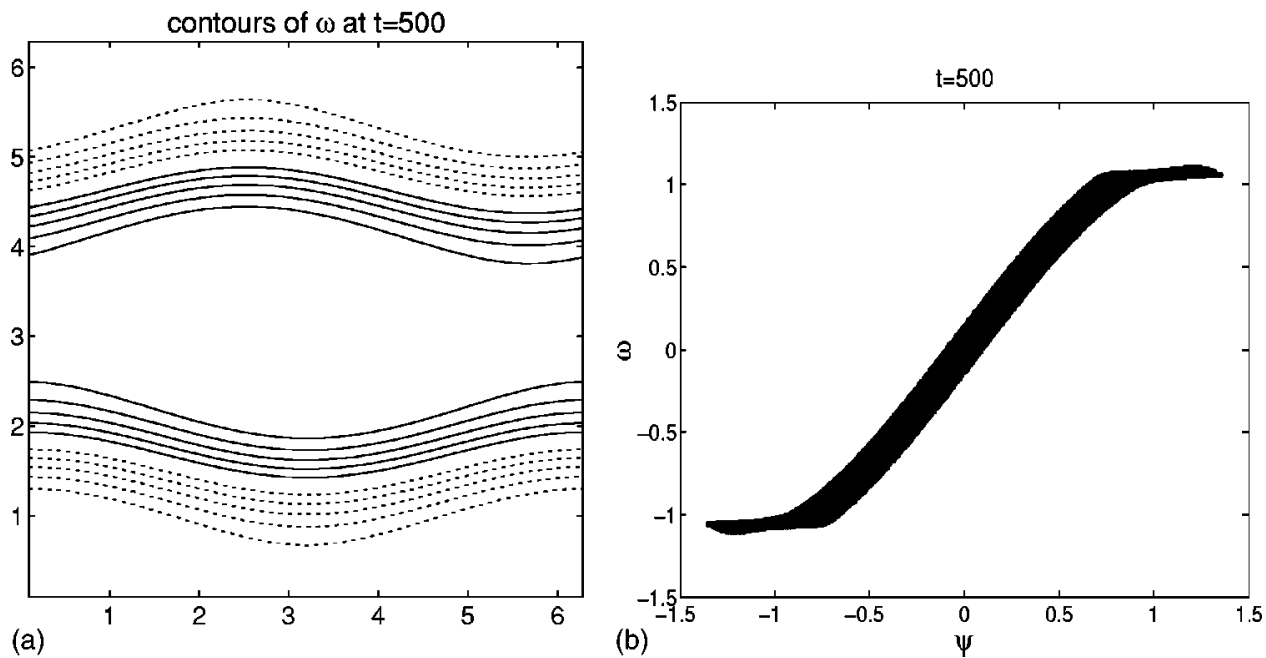

FIG. 5. In the late stage of the quadrupole to bar simulation (Ref. 2), a traveling wave appears and exists for a very long time: from $t=50$ to $t$ $=1000$. (a) shows one contour plot of the vorticity during this stage; (b) shows the corresponding $\omega$ - $\psi$ plot. The points in (b) cover a band of area that cannot be regarded as zero.

(a)

(b)

(1)

If the multivalued structure does not enclose any area $(S \cong 0)$, such as Fig. 2(c), it can also be considered that the final state has been reached. [Actually, Fig. 2(c) is not a good example: if we put some noise onto the weird quadrupole, and use it as the initial condition of a DNS run, the simulation will end up with a normal sense of the $\omega-\psi$ functional relation. Therefore, this weird quadrupole is in fact a local equilibrium state. However, we have to make our definition able to sort out similar situations with maximum entropies, which might appear in the future research.]

(2) If the multivalued structure does enclose some areas, but they are arranged clockwise and counterclockwise, and can cancel each other (again, $S$ $\cong 0$ ). It can also be regarded that the final state has been reached. (We will show an example in Sec. III B.)

(3) If the multivalued structure encloses some areas, but clockwise and counterclockwise parts of them cannot cancel each other, the final state has not been reached, and continuing calculations are needed. An extreme example is the state when the scatter points of $\omega-\psi$ are distributed across the whole plot, which normally happens when the simulation is started with some random initial condition [e.g., Fig. 4(c)].

With this judgment, those "fake" final states, such as the long-lived traveling wave in the patch quadrupole to the bar simulation (Fig. 5), can be easily sorted out.

\section{FURTHER STUDIES ON THE BAR FINAL STATE}

In YMC, we have studied the emergence of the bar final state in a way that a large amount of noise was added to an antisymmetric basic flow (the quadrupole solution) to break the symmetry of the basic flow. We decided to devote more efforts to finding other initial conditions leading to the bar quasistationary final state. It will be found later in this sec- tion that the appearance of the bar final state is not so accidental as might erroneously be concluded from the previous set of simulations.

The simulations are finished by the dynamical pseudospectral-code of the $2 \mathrm{D}$ NS equation, employing a resolution of $512 \times 512$ Fourier modes. The time step in all simulations is fixed at 0.0005 and determined by the Currant-Friedricks-Lewey (CFL) condition. The initial energy, using the normalization of

$$
E=\frac{1}{2} \frac{1}{(2 \pi)^{2}} \iint \omega \psi d x d y,
$$

is 0.5 . There is no hyperviscosity or small-scale smoothing of any kind in our simulations.

\section{A. Simulations by shrinking the size of patches in the initial vorticity field}

The first idea here is to start from the same quadrupolar patch initial condition as YMC, but distort the initial condition slightly in the following way: we shrink the patch size with a small amount and reposition the patches slightly (e.g., see the plots in the first row of Fig. 6). The symmetry of the basic flow has already been broken, and there is no need to add any noise to it [unlike what we did in Fig. 7(b) of YMC]. The Reynolds number is fixed at $1 / \nu=8000$. As can be seen from Fig. 6, we have performed two simulations with two different distorted quadrupolar initial conditions, with the patch size reduced by a factor of $7 / 8 \times 7 / 8=49 / 64$ compared with the patch size of the original quadrupole initial condition [see Fig. 7(a) in YMC]. Both runs clearly reveal the emergence of the bar final state. A similar set of simulations has been carried out, but now with the patch size even further reduced. In Fig. 7, we have shown the vorticity contour plots of runs with the patch size reduced by a factor of $3 / 4 \times 3 / 4=9 / 16$, and it is clear that no bar final state is found in this case.

We may recall that in Fig. 4 of YMC, statistical mechanical theories predict that for a doubly periodical domain, 

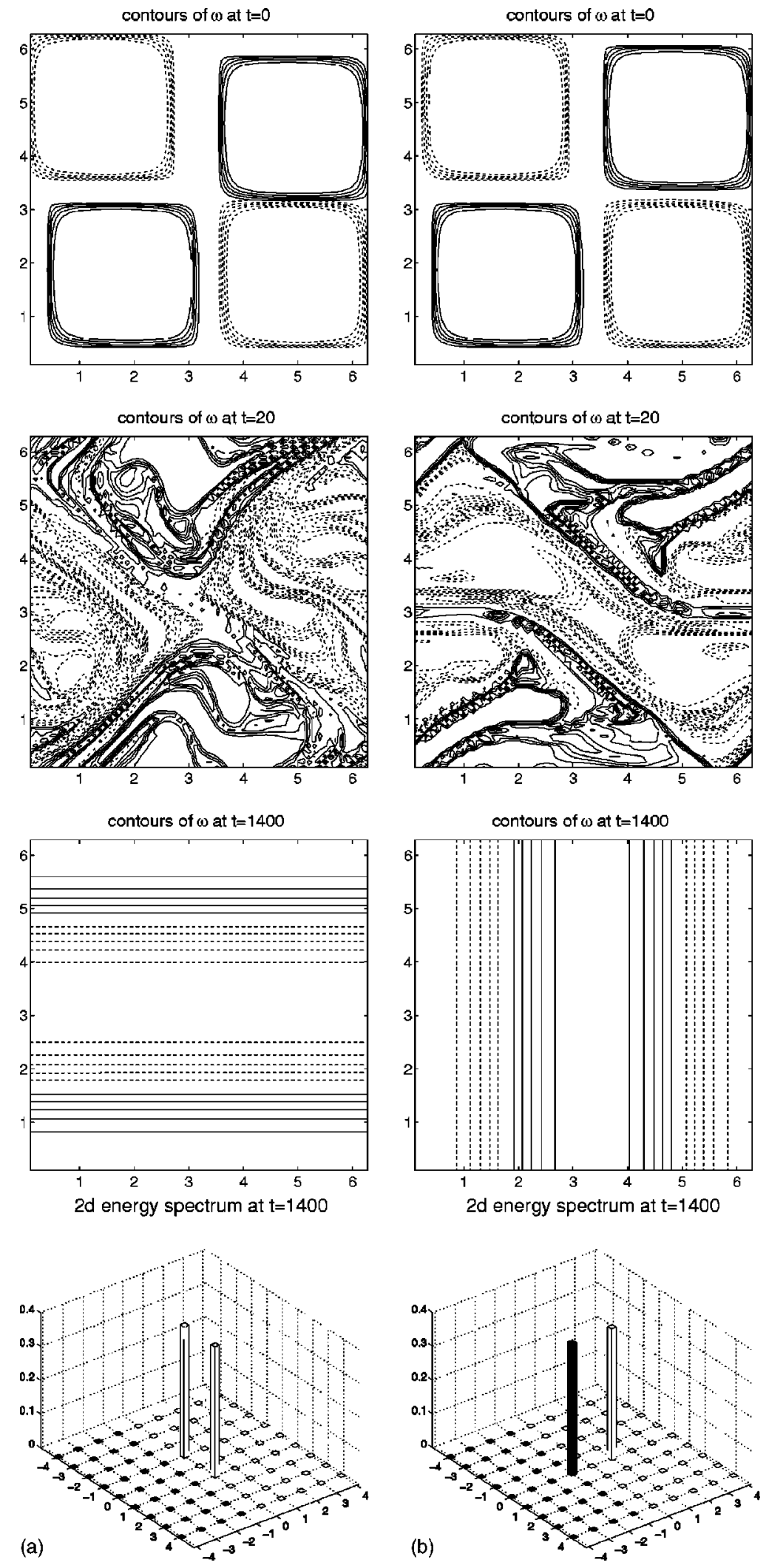

FIG 6. The first three rows are contours of constant vorticity for two runs with slightly different initial conditions in the left (a) and the right (b) column. In both runs, the initial patch sizes are reduced by a factor of $7 / 8 \times 7 / 8=49 / 64$, and the patches are displaced compared with the quadrupole initial condition shown in Fig. 7(a) of YMC. Pictures in the fourth row are modal energies of final states at low wave numbers for two runs. large patch vortices result in the bar final state and small patch vortices give rise to the dipole final state. In YMC, we only tested two extreme cases of theoretical results - the initial quadrupole solutions with the largest patches (Figs. 7-10 in YMC) and those with the smallest patches or point (Figs. 14-16 in YMC); no intermediate simulations can make the logic more complete. The four simulations in Figs. 6 and 7 of this paper provide a much more direct proof for our theoretical results of the statistical mechanics. Later in this section, it will be seen that if the size of the patch is shrunk by a factor even smaller than $9 / 16$, the numerical simulation will lead to the dipole final state. 

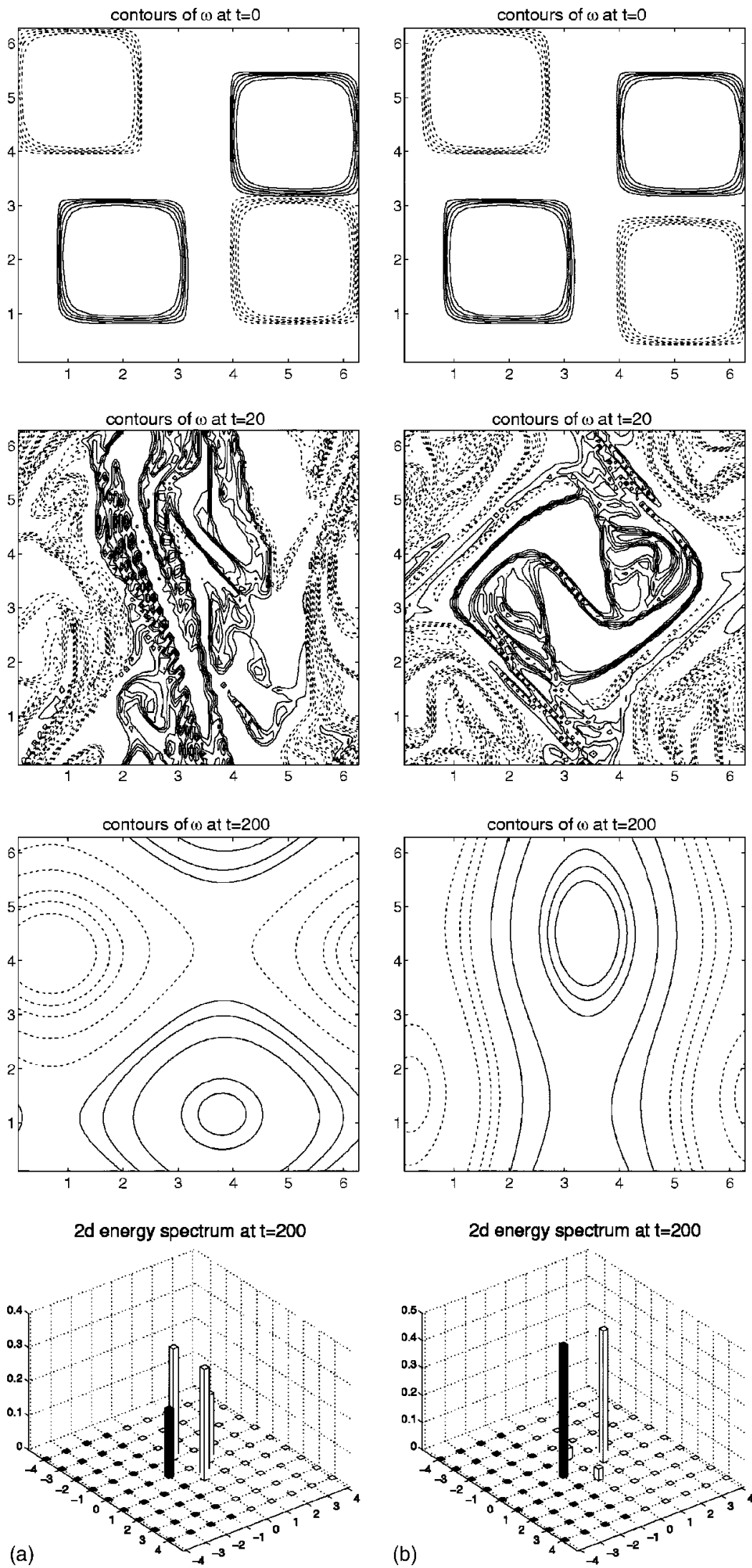

FIG. 7. The first three rows are contours of constant vorticity for two runs with slightly different initial conditions in the left (a) and the right (b) column. In both runs, the initial patch sizes are reduced by a factor of $3 / 4 \times 3 / 4=9 / 16$, which are somewhat larger reductions than initial conditions displayed in Fig. 6, and the patches are displaced with respect to the quadrupole initial condition shown in Fig. 7(a) of YMC. Pictures in the fourth row are modal energies of final states at low wave numbers for two runs.
One question that might be raised concerns the direction of the bar final state. As can be observed in Fig. 6, it can happen in the horizontal or vertical direction (either should occur with equal probability because of its symmetrical equivalence). However, the bar final state (with $2 \pi$ periodicity perpendicular to the flow direction) has never been observed in any other direction due to the lack of periodicity of such a solution. Note that a solution with periodicity less 

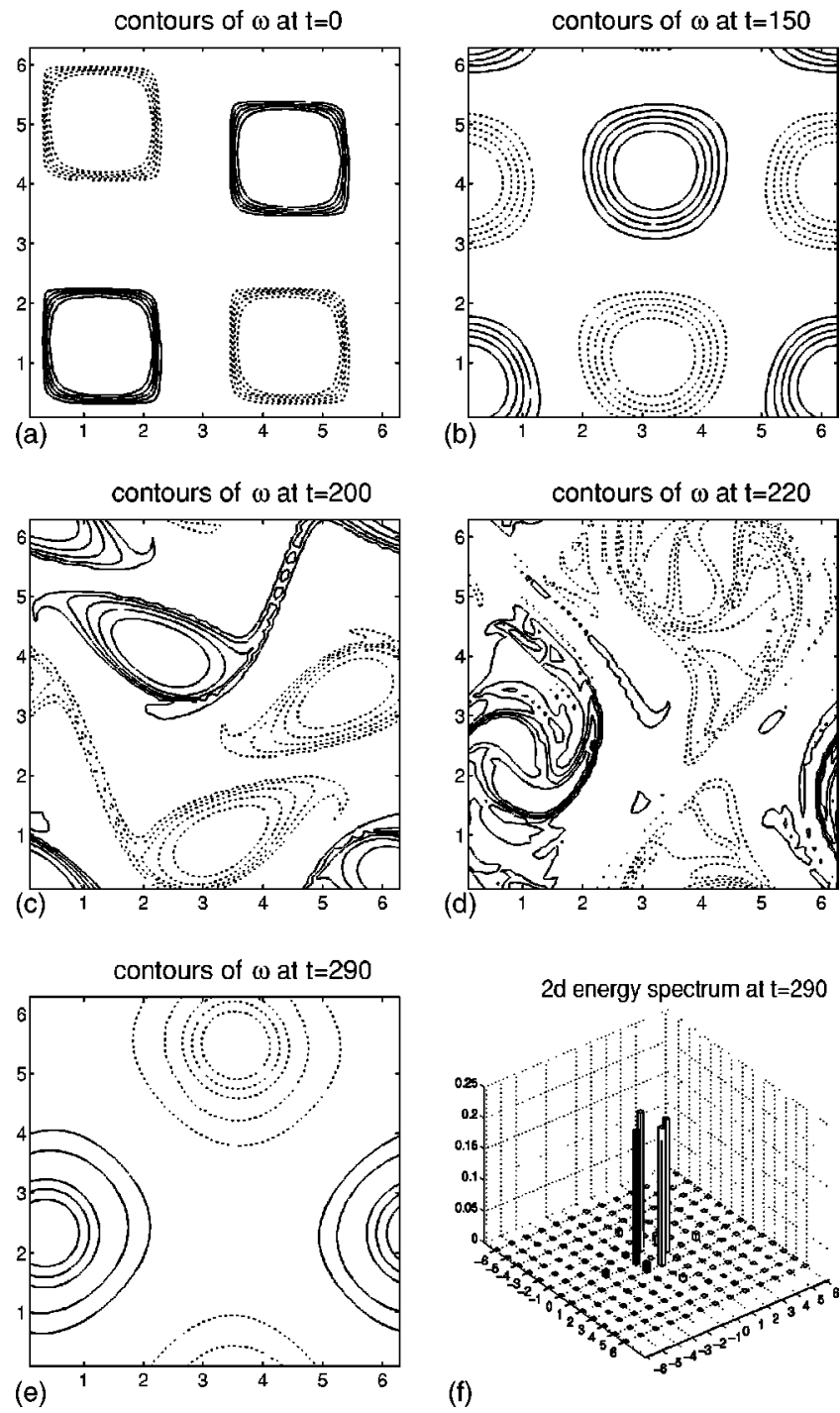

FIG. 8. In this run, the initial patch sizes are reduced by a factor of $5 / 8$ $\times 5 / 8=25 / 64$, which are even smaller than initial conditions displayed in Figs. 7(a)-7(e) are contour plots of vorticity and (f) is the plot of modal energies of the final state at low wave numbers.

than $2 \pi$ perpendicular to the flow direction enables a flow rotated with respect to the $x$ and $y$ direction (e.g., a direction of $45^{\circ}$ is needed for a bar solution with a $\sqrt{2} \pi$ periodicity).

Due to the inverse energy cascade phenomenon in 2D turbulence, most energy will be concentrated on the four lowest modes of wave numbers at final states,

$$
\vec{k}=\left(k_{x}, k_{y}\right)=(1,0),(-1,0),(0,1), \text { and }(0,-1) .
$$

Different combinations of these four modes dominate flow patterns at final states. Here are three kinds of possibilities:

(a) If energies are more or less equally distributed on these four modes, we will get the dipole [see the last vorticity contour plot in Fig. 5 of YMC or Fig. 8(f) of this paper];

(b) if energies are concentrated on either $(1,0),(-1,0)$ or $(0,1),(0,-1)$, then we will obtain bar final states (like Fig. 6 in this paper); (c) if energies are distributed on those modes unequally, then we will get a final state between dipole and bar [like Fig. 7 in this paper, especially Fig. 7(b)].

Several other runs with even smaller patch sizes were carried out, and their patch sizes are reduced by a factor of $5 / 8 \times 5 / 8=25 / 64,4 / 8 \times 4 / 8=1 / 4,3 / 8 \times 3 / 8=9 / 64,2 / 8$ $\times 2 / 8=1 / 16$, and $1 / 8 \times 1 / 8=1 / 64$, respectively. The Reynolds number is fixed at $1 / \nu=10000$. We only show the details of one of these simulations in the following.

In Fig. 8, the quadrupolar patches reduced by the factor of $5 / 8 \times 5 / 8=25 / 64$ are put asymmetrically to be used as the initial condition. The four patch vortices stay together in the flow field for a long time (from $t=0$ to $t \approx 180$ ), because they are far away from each other and there is almost no interaction among them. After $t \approx 190$, the flow begins to evolve into the final state. At $t \approx 290$, most energy is concentrated and more or less equally distributed on the four lowest modes of wave number [Fig. 8(f)]: $(1,0),(-1,0),(0,1)$, and $(0,-1)$. The functional $\omega-\psi$ relation is also discovered at the same time.

It should be realized that the size of the patches is not the only factor affecting the final energy distribution on the four lowest modes (these four modes determine whether the final state is dipole or bar). How to arrange the initial shrunk patches also takes effect, and there are many choices to achieve it. For example, although the two simulations in Fig. 7 have the patches with the same size, they show very different energy distributions at the final state (see the last row of Fig. 7). It is quite reasonable that the final state is very sensitive to the initial condition because the problem we are dealing with is a nonlinear one with a long-time evolution. Anyway, it still looks mysterious to us what threshold size might result in the dipole; and it might be very interesting to explore this topic in the future.

In Fig. 7 , both runs reach the final states at $t \approx 200$, which took shorter time than the run in Fig. 8. For the simulations started with even smaller quadrupolar patches than Fig. 8(a), it took longer time to get final states. The extreme case is the quadrupolar point vorticity initial field (when the size of patch is reduced to almost zero). The evolution of such a simulation is described in Fig. 7 of Ref. 2, where some random noise is added to accelerate the process, otherwise, it might take a very long time to get the final state.

\section{B. The "slanting bar" to bar simulation}

Another set of simulations with initial conditions leading to bar final states has been conducted. The initial condition is based on the slanting bar solution already referred to in the preceding section $(\sqrt{2} \pi$ periodicity), where a certain amount of noise is added to break the symmetry. We let the flow evolve and as shown in Fig. 9, the bar final state is obtained eventually. The Reynolds number in this simulation is fixed at $1 / \nu=8000$.

Attention should be drawn to the $\omega-\psi$ scatter plot obtained at the end of the simulation. It is similar to the scatter plot obtained for the bar solution in Fig. 9 of YMC, but some subtle differences can be observed. When considered in more 

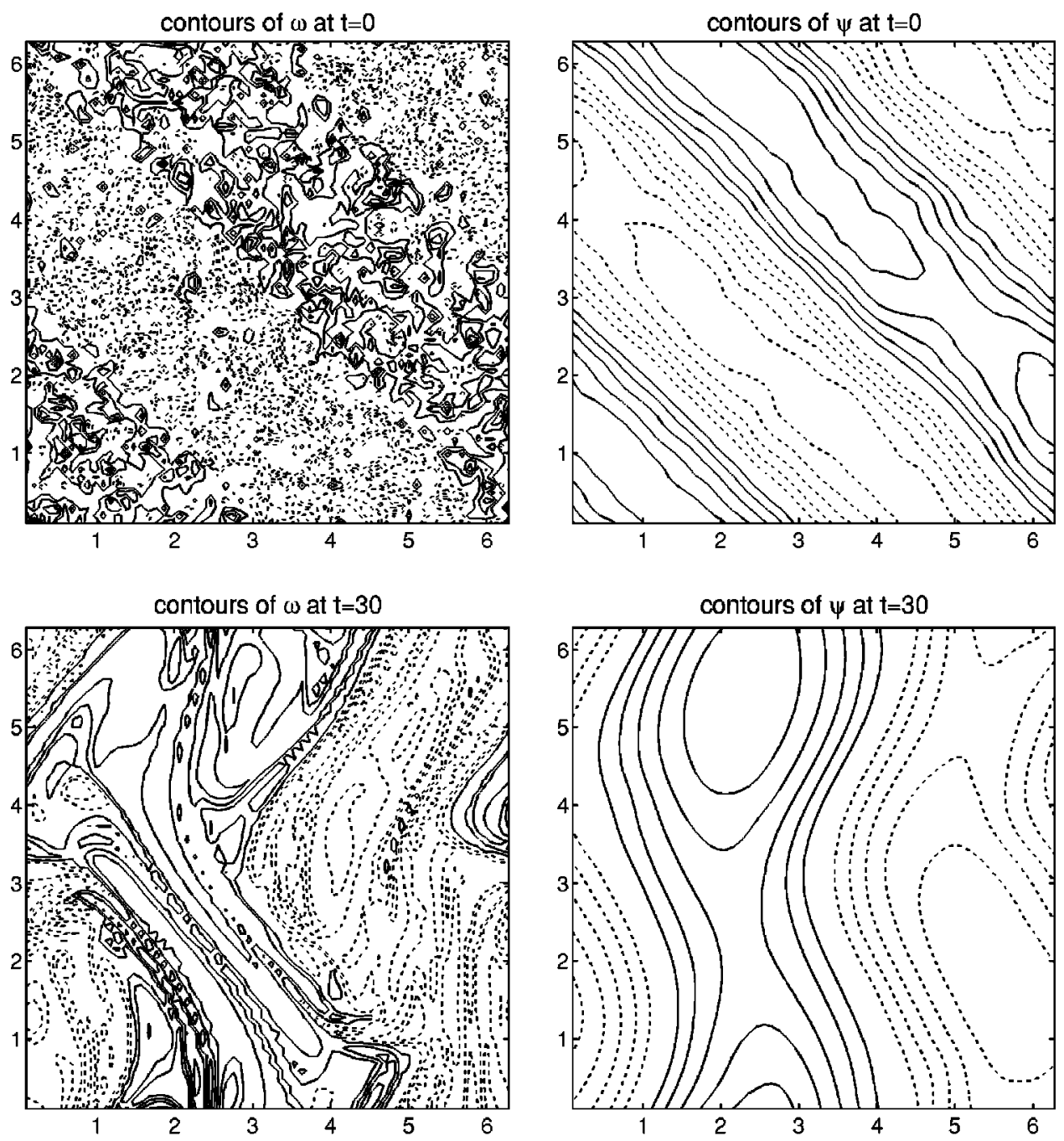

FIG. 9. Contours of constant vorticity (left column) and constant stream function (right column) at three different times for the run with a new initial condition leading to the "bar" final state. The arrow line in the last figure of the right column is used to mark the flow field and calculate the effective area $S$ in Fig. 10.
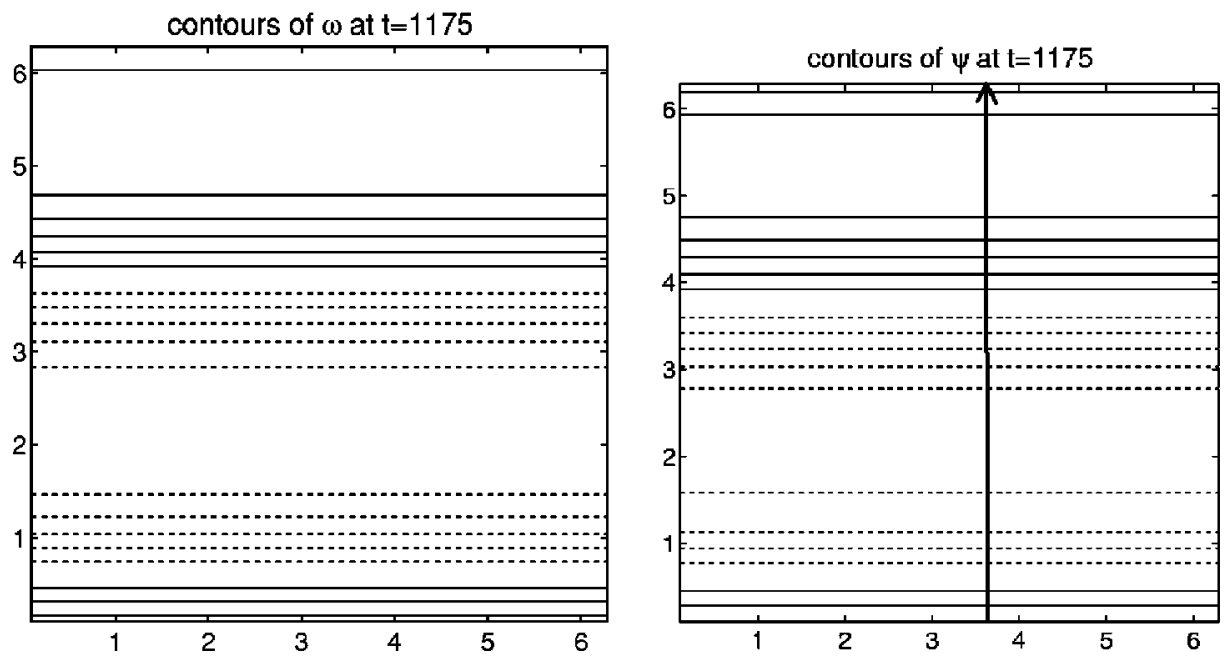

details, the scatter plot is in fact a double-valued structure. However, this double-valued structure is different from the open line in Fig. 2(c), and this structure actually encloses some areas. We may recall the discussion in Sec. II: if those areas cannot cancel each other, namely, $S \neq 0$, it may mean that the final state has not been reached and continuing calculation is required. However, with a close examination of the $\omega-\psi$ scatter plot, it will be found that the region covered by scatter points is actually reentrant, and thus $S \approx 0$.

This procedure can be fulfilled by drawing a line from the bottom to the top of the contour plot of $\psi$ (see the arrow line at the last figure in the right column of Fig. 9). It is not necessary to draw a loop [like what we did in Fig. 3(a)] in this case, because we are dealing with the doubly periodical 


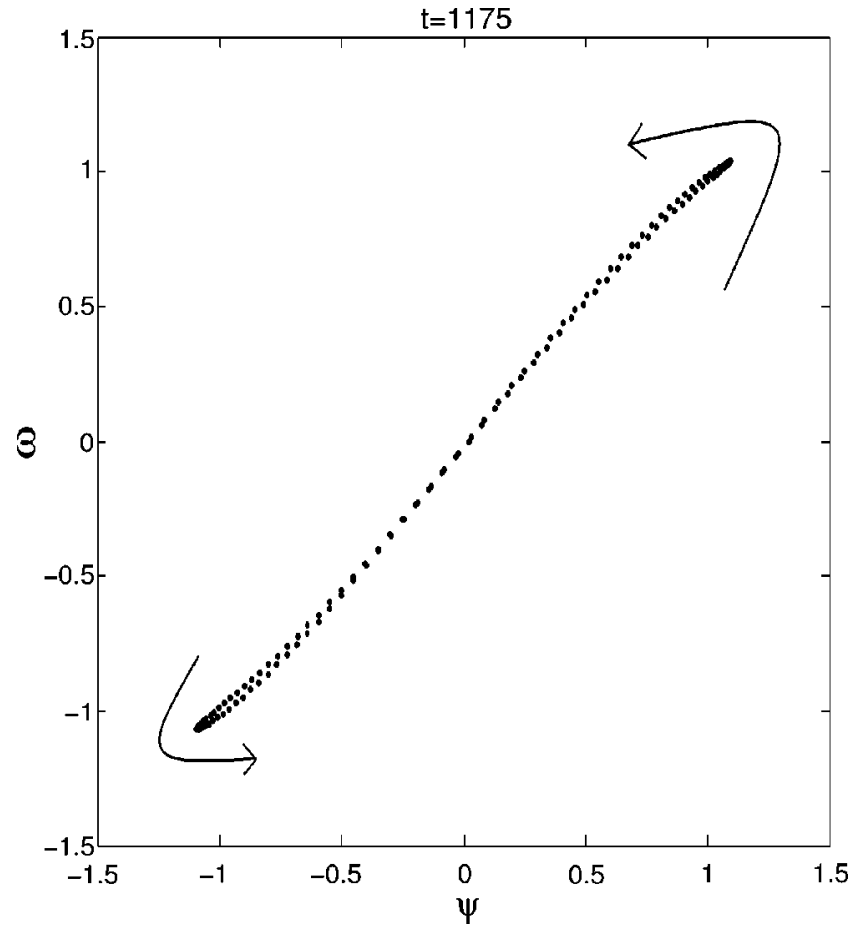

FIG. 10. The $\omega-\psi$ scatter plot for the run shown in Fig. 9. The two ends of the plot are actually two loops. The two arrows indicate the orientations of these two loops, which are obtained by the location of the corresponding points along the arrow line in the last plot of Fig. 9.

condition, and a straight line connecting two opposite boundaries is already enough to make a loop. At the late stage of this simulation, the flow field is essentially one dimensional. It is possible to gather all the information by studying this straight line. As indicated in Fig. 10, the corresponding points in $\omega-\psi$ plot form a clockwise region and a counterclockwise region, which can almost cancel each other [see Eq. (7)]. As a result, the absolute value of the effective area $S$ is very small-the final state has been reached.

This simulation illustrates one special case covered by our final state definition (see the second last paragraph in Sec. II B). Again, statistical mechanics cannot explain this double-valued structure due to the less "turbulent" initial condition.

\section{DISCUSSIONS AND CONCLUSIONS}

In this paper, we have shown two kinds of double-valued $\omega-\psi$ structures from direct numerical simulations [Fig. 2(c) and 10]. It should be noticed that in the process of deriving the formulas of statistical theories, Lagrange multipliers methods are always applied to get the equilibrium states under certain constraints (e.g., see Ref. 29). Lagrange multipliers methods adopt the assumption that all involved functions are differentiable. Hence, the double-valued structures, which have at least one singular point without a derivative, can never be achieved. So the functional relation $\omega=f(\psi)$ is a very basic assumption in 2D statistical mechanics, and the double-valued $\omega-\psi$ structure can never be well interpreted as long as we stick to statistical mechanics.
Furthermore, from the viewpoint of pure mathematics, $J(\omega, \psi)=0$ [Eq. (3)] can easily be obtained if we know there is a functional relation $\omega=f(\psi)$. On the other hand, we cannot get $\omega=f(\psi)$ if we only know $J(\omega, \psi)=0$ without any other assumption.

Luckily, if we take the vorticity field of those doublevalued $\omega-\psi$ structures as the combination of two subdomains (in the case of Fig. 2, the vorticity field can be divided by making each subdomain have one positive and one negative vortex, respectively), we can still get the functional $\omega-\psi$ relation in each subdomain. Within those subdomains, statistical mechanics can still manage to give an explanation.

To sum up, we try to give a definition of the final state of 2D decaying turbulence in this paper. The definition using the effective area $S$ in the $\omega-\psi$ space is more general than the ordinary functional relation and can cover all existing results. Some DNS results, which further confirm the predictive power of the statistical mechanics, are also found.

Like what was indicated in the left column of Fig. 10 of YMC, both simulations in Fig. 6 of this paper are started from initial conditions in which most energies are concentrated in few narrow-band low wave numbers. All existing numerical results, which lead to the bar final state with the $\omega-\psi$ functional relation and therefore verify the patch theory, have similar initial conditions. On the other hand, these initial conditions are less turbulent than the broad band wave number states (e.g., the flow field shown in the first row of Fig. 5 of YMC). Sometimes they lead to some results predicted by the patch theory (Fig. 9 in YMC), but sometimes they also result in some weird states (Figs. 2 and 10) that no existing statistical mechanics can explain. Because of the nonlinear behavior of 2D turbulence, it is almost impossible to tell when those "weird final states" will appear from the information of the initial condition.

Finally, for the integrality of studies on the patch and point theory in the statistical mechanics, we should not only connect the statistical theory with numerical simulations (in YMC and this paper), but also connect the theory with experiments.

(1) For the patch theory, so far as to our knowledge, there is no experiment setup that can produce flat vortices, which are illustrated in our numerical simulations. It would be exciting to see such kind of experiments in the future.

(2) For the point theory, it is easier to produce random point vortices in the laboratory, but our numerical code and theory results are only dealing with the doubly periodic domain for the time being. It is easier to achieve high resolutions and perform high Reynolds number simulations by doing this, but it is also difficult to find any experimental comparison. In the next step of this research, we will try to connect the theory with more complex boundaries (e.g., the no-slip boundary), for which some laboratory proofs can be easily found. 


\section{ACKNOWLEDGMENTS}

The author would like to thank Professor G. J. F. van Heijst who pointed out flaws ranging from minor to major in the earlier drafts of this paper. Sincere thanks also go to Professor D. C. Montgomery and Dr. H. J. H. Clercx for making corrections in the corresponding part of the author's Ph.D. thesis. The author wants to thank Dr. R. R. Trieling, Professor E. van Groesen, Professor T. J. Schep, Professor Annick Pouquet, Professor W. van de Water, Professor F. W. Sluijter, Professor Zhiming Chen, Professor Li Yuan, and Professor Linbo Zhang for useful discussions and invaluable help. Numerical simulations in this paper were fulfilled on the SGI Origin 3800 at SARA Supercomputing Center in Amsterdam and the Legend DeepComp 1800 at LSEC, Academy of Mathematics and System Science in Beijing.

${ }^{1}$ Z. Yin, D. C. Montgomery, and H. J. H. Clercx, "Alternative statisticalmechanical descriptions of decaying two-dimensional turbulence in terms of 'patches' and 'points'," Phys. Fluids 15, 1937 (2003) (referred to as YMC).

${ }^{2}$ Z. Yin, H. J. H. Clercx, and D. C. Montgomery, "An easily implemented task-based parallel scheme for the Fourier pseudospectral solver applied to 2D Navier-Stokes turbulence," Comput. Fluids 33, 509 (2004).

${ }^{3}$ G. R. Joyce and D. Montgomery, "Negative temperature states for a twodimensional guiding-centre plasma," J. Plasma Phys. 10, 107 (1973).

${ }^{4}$ D. Montgomery and G. R. Joyce, "Statistical mechanics of negative temperature states," Phys. Fluids 17, 1139 (1974).

${ }^{5}$ L. Onsager, "Statistical hydrodynamics," Nuovo Cimento, Suppl. 6, 279 (1949).

${ }^{6}$ B. E. McDonald, "Numerical calculation of nonunique solutions of a twodimensional sinh-Poisson equation," J. Comput. Phys. 16, 630 (1974).

${ }^{7}$ D. L. Book, S. Fisher, and B. E. McDonald, "Steady-state distributions of interacting discrete vortices," Phys. Rev. Lett. 34, 4 (1975).

${ }^{8}$ Y. B. Pointin and T. S. Lundgren, "Statistical mechanics of twodimensional vortices in a bounded container," Phys. Fluids 19, 1459 (1976).

${ }^{9}$ J. H. Williamson, "Statistical mechanics of a guiding-centre plasma," J. Plasma Phys. 17, 85 (1977).

${ }^{10}$ A. C. Ting, H. H. Chen, and Y. C. Lee, "Exact solutions of nonlinear boundary value problem: The vortices of the two-dimensional sinhPoisson equation," Physica D 26, 37 (1987).

${ }^{11}$ R. A. Smith, "Phase transition behavior in a negative-temperature guidingcenter plasma," Phys. Rev. Lett. 63, 1479 (1989).

${ }^{12}$ R. A. Smith and T. O'Neil, "Nonaxisymmetric thermal equilibria of a cylindrically-bounded guiding-center plasma or discrete vortex system," Phys. Fluids B 2, 2961 (1990).

${ }^{13}$ R. A. Smith, "Maximization of vortex entropy as an organizing principle of intermittent, decaying, two-dimensional turbulence," Phys. Rev. A 43, 1126 (1991).

${ }^{14}$ L. J. Campbell and K. O'Neil, "Statistics of 2D point vortices and high energy vortex states," J. Stat. Phys. 65, 495 (1991).

${ }^{15}$ R. H. Kraichnan and D. Montgomery, "Two-dimensional turbulence," Rep. Prog. Phys. 43, 547 (1980).

${ }^{16}$ M. K.-H. Kiessling, "Statistical mechanics of classical particles with logarithmic interactions," Commun. Pure Appl. Math. 46, 2108 (1993).

${ }^{17}$ G. L. Eyink and H. Spohn, "Negative temperature states and large-scale, long-lived vortices in two-dimensional turbulence," J. Stat. Phys. 70, 833 (1993).

${ }^{18}$ A. J. Chorin, Vorticity and Turbulence (Springer, New York, 1994).

${ }^{19}$ K. W. Chow, N. W. M. Mo, R. C. K. Leung, and S. K. Tang, "Inviscid two dimensional vortex dynamics and a soliton expansion of the sinh-Poisson equation," Phys. Fluids 10, 1111 (1998).

${ }^{20}$ B. N. Kuvshinov and T. J. Schep, "Double-periodic arrays of vortices," Phys. Fluids 12, 3282 (2000)

${ }^{21}$ F. Spineanu and M. Vlad, "Self-duality of the asymptotic relaxation states of fluids and plasmas," Phys. Rev. E 67, 046309 (2003).

${ }^{22}$ K. W. Chow, S. C. Tsang, and C. C. Mak, "Another exact solution for two-dimensional, inviscid sinh-Poisson vortex arrays," Phys. Fluids 15, 2437 (2003)

${ }^{23}$ R. Robert and J. Sommeria, "Statistical equilibrium states for twodimensional flow," J. Fluid Mech. 229, 291 (1991).

${ }^{24}$ R. Robert and J. Sommeria, "Relaxation towards a statistical equilibrium in two-dimensional perfect fluid dynamics," Phys. Rev. Lett. 69, 2776 (1992).

${ }^{25} \mathrm{P}$. H. Chavanis and J. Sommeria, "Classification of self-organized vortices in two-dimensional turbulence: The case of a bounded domain," J. Fluid Mech. 314, 267 (1996).

${ }^{26} \mathrm{P}$. H. Chavanis and J. Sommeria, "Classification of robust isolated vortices in two-dimensional hydrodynamics," J. Fluid Mech. 356, 259 (1998).

${ }^{27}$ J. Miller, P. C. Weichman, and M. C. Cross, "Statistical mechanics, Euler's equation, and Jupiter's red spot," Phys. Rev. A 45, 2328 (1992), and references therein.

${ }^{28}$ H. Brands, S. R. Maassen, and H. J. H. Clercx, "Statistical mechanical predictions and Navier-Stokes dynamics of two-dimensional flows on a bounded domain," Phys. Rev. E 60, 2864 (1999).

${ }^{29}$ D. Lynden-Bell, "Statistical mechanics of violent relaxation in stellar systems," Mon. Not. R. Astron. Soc. 136, 101 (1967).

${ }^{30} \mathrm{E}$. Segre and S. Kida, "Late states of incompressible 2D decaying vorticity field," Fluid Dyn. Res. 23, 89 (1998).

${ }^{31}$ H. Lamb, Hydrodynamics, 6th ed. (Cambridge University Press, Cambridge, 1932).

${ }^{32}$ J. H. G. M. van Geffen and G. J. F. van Heijst, "Viscous evolution of 2D dipolar vortices," Fluid Dyn. Res. 22, 191 (1998).

${ }^{33}$ W. H. Matthaeus, W. T. Stribling, D. Martinez, S. Oughton, and D. Montgomery, "Selective decay and coherent vortices in two-dimensional incompressible turbulence," Phys. Rev. Lett. 66, 2731 (1991).

${ }^{34}$ W. H. Matthaeus, W. T. Stribling, D. Martinez, S. Oughton, and D. Montgomery, "Decaying two-dimensional turbulence at very long times," Physica D 51, 531 (1991).

${ }^{35}$ D. Montgomery, W. H. Matthaeus, W. T. Stribling, D. Martinez, and S. Oughton, "Relaxation in two dimensions and the 'sinh-Poisson' equation," Phys. Fluids A 4, 3 (1992).

${ }^{36}$ Such relation, which is also called "the multivalued function" sometimes, is different from the ordinary definition of the function because its derivatives cannot be defined. In Eq. (5), the relation we use to derive $J(\omega, \psi)$ $=0$ is the normal sense of function, which is so-called one-to-one or manyto-one function [i.e., for any value of $x$, we can only get one value of $y$ through relation $y=f(x)]$. In this paper, the ordinary definition is always adopted whenever the word "function" is referred to.

${ }^{37}$ P. L. Read, P. B. Rhines, and A. A. White, "Geostrophic scatter diagrams and potential vorticity dynamics," J. Atmos. Sci. 43, 3226 (1986).

${ }^{38}$ B. M. Boubnov, S. B. Dalziel, and P. F. Linden, "Source-sink turbulence in a stratified fluid," J. Fluid Mech. 261, 273 (1994).

${ }^{39}$ F. de Rooij, P. F. Linden, and S. B. Dalziel, "Experimental investigations of quasi-two-dimensional vortices in a stratified fluid with source-sink forcing," J. Fluid Mech. 383, 249 (1999). 